\title{
Designing of Counters using JK Flip-Flop in Quantum Dot Cellular Automata
}

\author{
E. Prashanthi, H. Bhagya laxmi
}

\begin{abstract}
In the post-CMOS condition an enormous development was played by the qca progress. As the CMOS improvement has a scaling tangle in focal nanometer drives, the best choice for CMOS progress was a quantum-spot cell automata progress. It has the combined thought of quantum mechanics \&cell automata. QCA (quantum bit cell automata) was a quantum wonder that gives another computational stage to structure electronic circuits using quantum contacts \& move information at nano level as a substitution of standard CMOS based movement. In standard CMOS improvement, transistors are used to make any robotized circuit yet in QCA, qca cells are used to make moved circuits \&wire. In this paper, we proposed the breeze around counters using JK flip-flop. 2-piece \&4-piece counters are executed using the QCA Technology. The perspective was filtered through \& the solace of the circuit was surrendered by using $Q C A$ sketcher contraption.
\end{abstract}

Key words: quantum spot cell automata, counters, JK flip-flop, QCA organizer gadget.

\section{INTRODUCTION}

The circumstance of the pushed business has changed basically due to the quick improvement in the progression. Generally CMOS excessively perceived indeed understood progression was applied in the structure of current actuated clarification circuits. As displayed by Gordon Moore's aching, the standard CMOS based contraptions advanced from micron to imperative submicron \& some time later to nanometer over late decades. As the transistor size was diminishing, the improvement was going toward some physical \& scaling obstructs, for instance, tunneling streams, sub-edge spillage, quantum impacts, influence cost, to interconnect delay. So elective evaluations have been done to build up another progress which can allow the improvement of the device thickness.

Craig Lent el at. Proposed another physical execution of an automation framework using the quantum spot cells in 1993. It promptly got reputation \& made in 1997 . So now reliably's QCA (quantum contact cell automata) was one of the charming subjects \& was used for use of nano-scale circuits. In QCA, the clarification states are not managed as voltage levels, yet rather the circumstance of individual electron was considered. It relies on coulomb revultion between electrons which empowers information to be moved

beginning with one cell then onto the join by advancement of polarization passed on instead of electrical stream. Information was coded using cells holding only 2 stable

Revised Manuscript Received on December 12, 2019

E.Prashanthi, Assistant professor (ECE), Malla Reddy Engineering College for Women, Telanagana, India, prashanthiede@gmail.com

H. Bhagya Laxmi, Assistant professor (ECE), Malla Reddy Engineering College for Women, Telanagana, India, Bhagya.hash@gmail.com states used to address moved characteristics. Near cells impact each other like the domino chain. Thusly the area required by the QCA cell was low \& the trading speed of the cell was enthusiastic. All things considered the power use was extraordinarily low. So the inclinations over CMOS join lesser deferment, high thickness circuits \& low power use. Since QCA cells move information by the advancement of charmed charge rather than stream of current.

\section{BASICS OF QCA}

QCA cell was the central bit of this headway merges the nano-scale square with four charged compartments that are called quantum spots in its corners \& two adaptable electrons. a electrons will by an live corner to corner to each other because of air central electrostatic repulsive power between am. ay will by $\&$ large keep a most remote partition between each other in a square model structure \& produce two novel states watching out for system for deduction 0 ( tended to as $\mathrm{p}=-1)$ \& reason 1 ( tended to as $\mathrm{p}=+1$ ) \& by engineering ase cells, system for deduction limits are done.

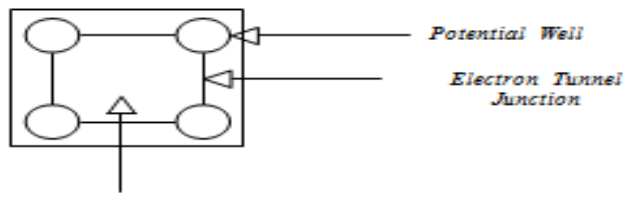

Cell Substrate

Fig: 1 Anatomy of QCA cell

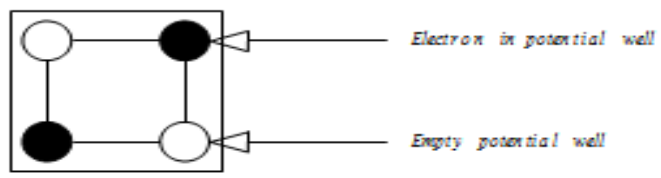

Fig: 2 Electrons in potential we1

There are 2 separation conditions in QCA cell that can-be used to address twofold side interests $0 \& 1$ as showed up in figure: 3

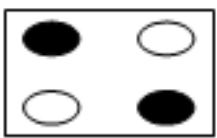

Binaryo $P=-1$

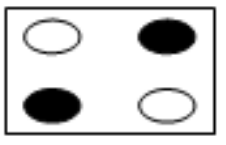

Binary 1

$P=+1$
Fig: 3 Binary depictions of QCA cells.

QCA wire was depended upon to transmit signals. a wire was made by working by building two or three cells in a line. In a wire an electron terribleness saw by a columbic

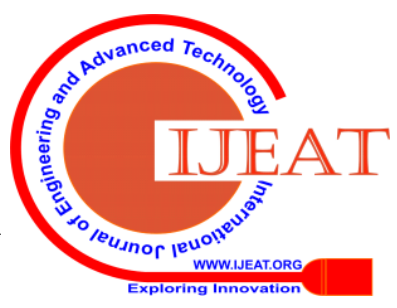


correspondence between one cell \&its neighbor will change polarization of a going with cell (neighbor cell). This approach will proceed down a length of a wire. To keep up an improvement of data sufficiently through a wires, we should give a best clock signal. In a standard QCA wire a relative polarization state was passed on which was appeared in fig: 4

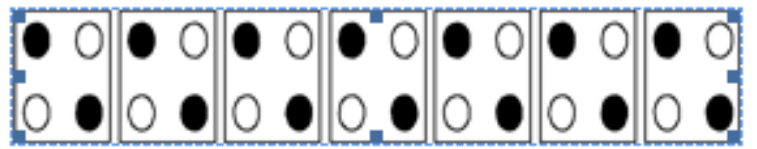

Fig: 4 QCA wire using ordinary Q Qैं

\section{(A) QCA INVERTER:}

a inversion was a most principal advancement of cutting edge contraptions. a QCA inverter was framed by sorting out cells corner to corner from each other.

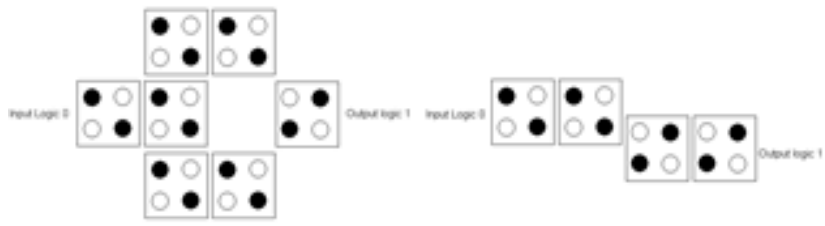

Fig: 5 Two different topologies of QCA inverter

\section{(B) QCA MAJORITY GATE:}

The basic structure of overwhelming part entry contains least five QCA cells to address it. Four of am are in four stand-disconnected ways making amselves at an edge of 90 degree with a closest neighboring cells on a substrate \&are was one cell which was in explanation behind relationship of am as appeared in fig: 6

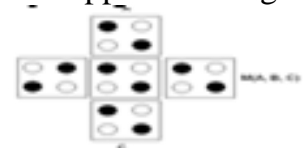

(a)

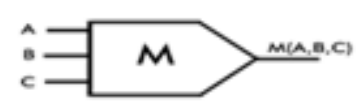

(b)
Fig: 6 (a) QCA cell arrangement (b) symbol for majority gate

The Boolean expression was

$$
\mathrm{M}(\mathrm{A}, \mathrm{B}, \mathrm{P})=\mathrm{A} \cdot \mathrm{B} \cdot \mathrm{P}+\mathrm{A} \cdot \mathrm{P}+\mathrm{B} \cdot \mathrm{P}
$$

\section{(C) CLOCKING:}

Clock was used to crush a tunneling hinders between a reaching quantum spots. In this way to keep up a basic tolerable ways from a information from getting lost, a information sign ought to have been restored once in a while during a transmission strategy.

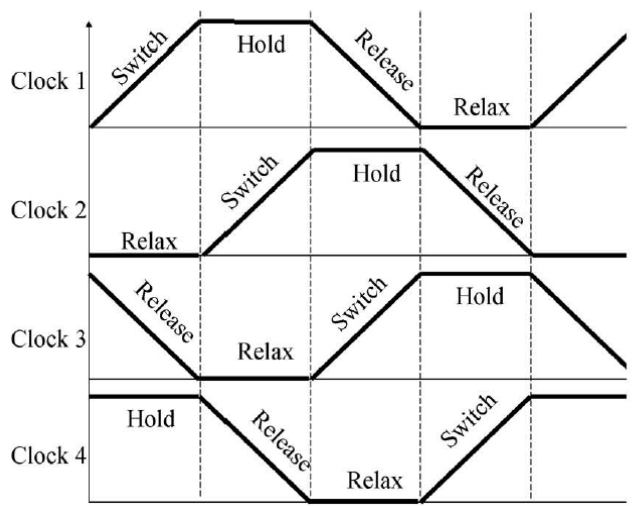

Fig: 7 four clock zones in QCA

\section{PROPOSED METHODOLOGY}

The instigated contraptions are confined into 2 classes: combinational \& dynamic. Boolean strategy for speculation limits which are used in regular CMOS improvement, at any rate in powerful circuits its existence table was seen from which a Boolean explanations were settled.

JK flip-flop:

The JK Flip-flop resembles a SR Flip-flop yet are was no change in state when a $\mathrm{J} \& \mathrm{~K}$ inputs are both LOW. This sensible JK flip-flop was regularly used among all other flip-flop structures \& was considered as a broad flip-flop circuit. a data sources $\mathbf{J} \& \mathrm{~K}$ are named after its maker jack kilby.Fig:8 address a JK flip-flop sifted through using lion's offer entryways \& its strategy in QCA originator instrument.

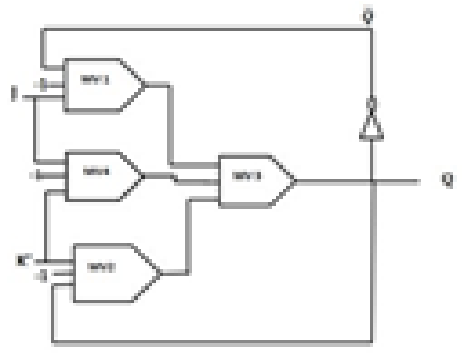

(a) Block diagram

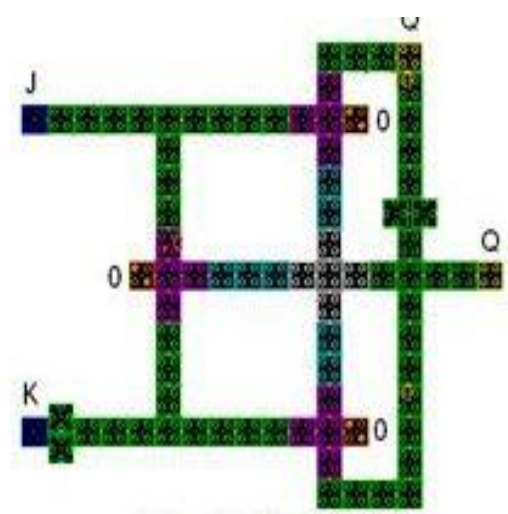

(b) Layout using QCA 


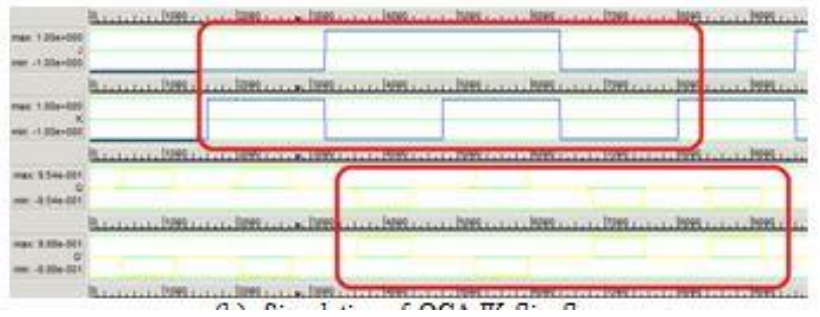

(c) Simulation result using QCA

Fig: 8(a) JK flip-flop circuit using winning part

entryways (b) gathering (c) age result using qca

Counter was a back to back circuit \& a fundamental piece of front line structures. It fuses set flip-flops related in a sensible manner to check a course of action of data beats. Here a counter was recognized with a persistent relationship of JK flip-flop in QCA using QCADesigner gadget which was showed up in underneath circuits. In this paper we are looking 2-piece, 3-piece \& 4-piece synchronous counter composed by using QCA progress.

\section{2-BIT COUNTER USING JK FLIP-FLOP:}

The 2-piece synchronous up counter showed up in Fig. 9 was worked by falling two a two obligations of JK flip-flop. Yield a gives a least essential bit of a 2-piece synchronous counter. To make a MSB, yield B must be improved every two cycles. Right when yield An was reason 0, yield B will hold, \& when yield An was premise 1, yield B will flip. Essentially, yield B was improved when yield a goes from procedure for deduction 1 to introduce 0 .

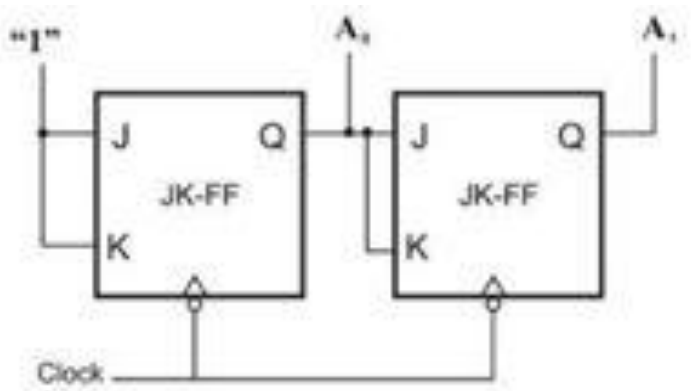

(a) Block diagram

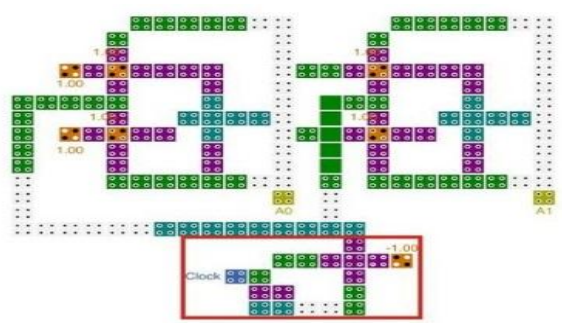

(b) Layout using QCA

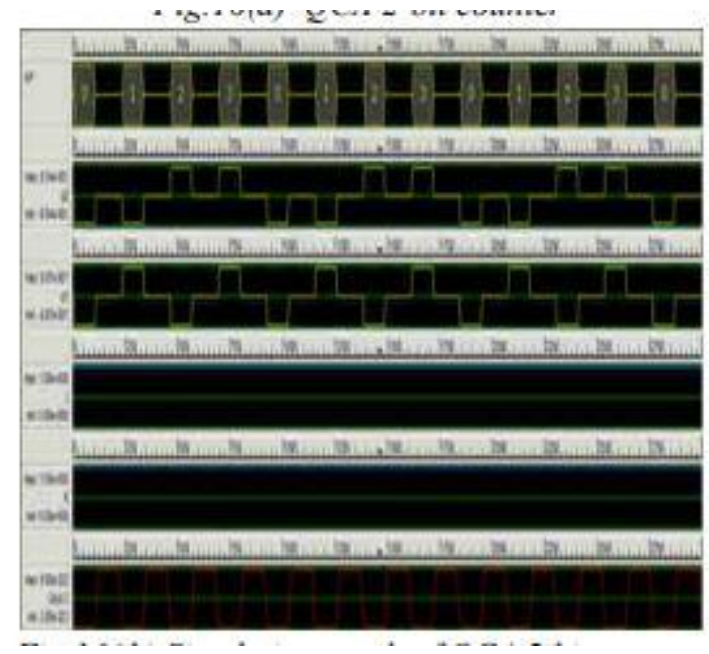

(c) Simulation result using QCA

Fig: 9 2-bit synchronous counter using JK flip-flop 3-BIT COUNTER USING JK FLIP-FLOP:

The below figure indicates the 3-Bit counter using JK flip-flop.

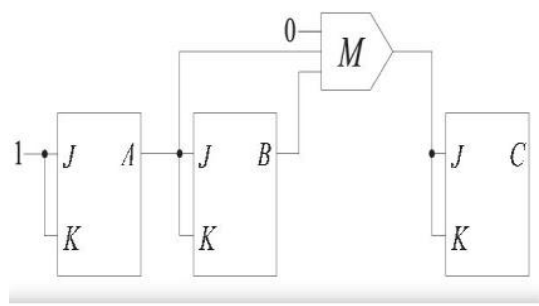

(a) Block diagram

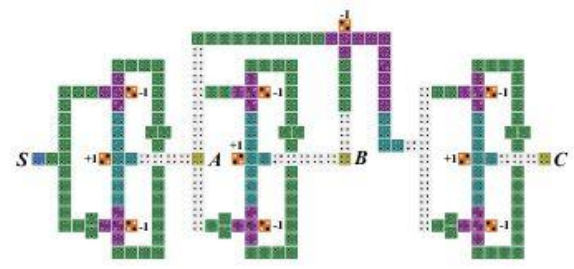

(b) Layout using QCA

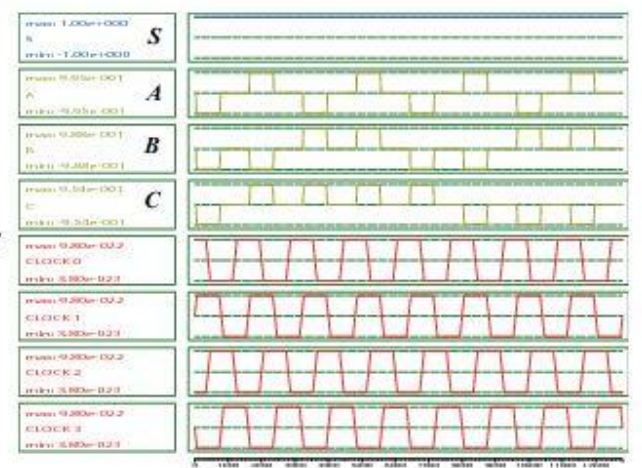

(c) Simulation result using QCA

Fig: 10 3-bit synchronous counter using JK flip-flop

\section{4-BIT COUNTER USING JK FLIP-FLOP:}

The outcome was a four-piece synchronous counter. Each higher referencing flip-flops are set up to flip i.e., both J \&K inputs "high" if a Q yields of all past flip-flops are "high." Otherwise, a J \&K commitments for that flip-lemon will both be "low," setting it into a "get" mode where it will keep up its

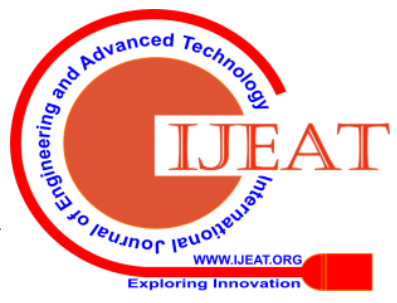


present yield state at a going with clock beat. Since a first (LSB) flip-flop needs to flip at each clock beat, its $\mathrm{J} \& \mathrm{~K}$ inputs are will be "high" perseveringly. a going with flip-flop need on an essential level "see" that a vital flip-disorder's Q yield was high to be set up to flip.

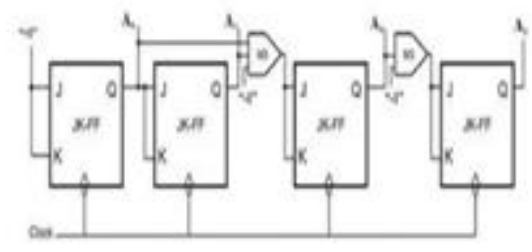

(a) Block diagram

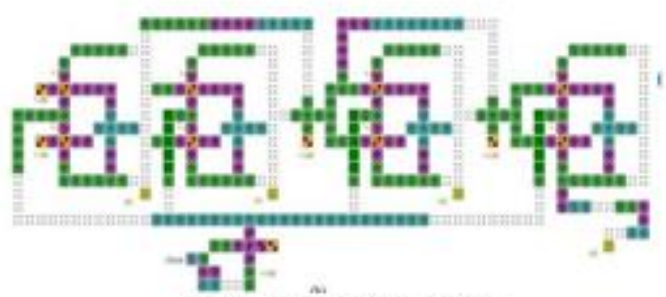

(b) Layout using QCA

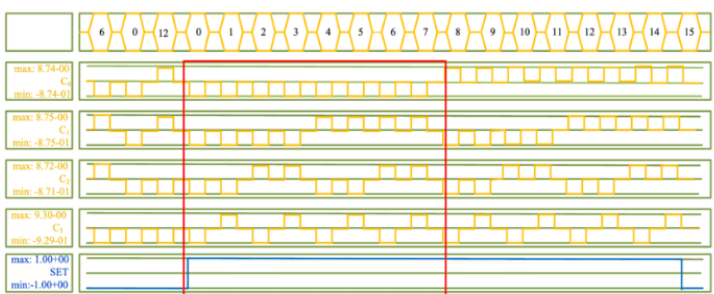

(c) Simulation result using QCA

Fig: 11 4-bit synchronous counter using JK flip-flop

TABLE: I counter design: No. of cells \& area occupied

\begin{tabular}{|c|c|c|c|}
\hline counters & cells & Area(um2) & \multicolumn{1}{|c|}{ Delay } \\
\hline $\begin{array}{c}\text { 2-bit } \\
\text { synchronous } \\
\text { counter }\end{array}$ & 240 & 0.26 & $\begin{array}{c}2 \text { clock } \\
\text { cycles }\end{array}$ \\
\hline $\begin{array}{c}\text { 3-bit } \\
\text { synchronous } \\
\text { counter }\end{array}$ & 428 & 0.48 & $\begin{array}{c}2 \text { clock } \\
\text { cycles }\end{array}$ \\
\hline $\begin{array}{c}\text { 4-bit } \\
\text { synchronous } \\
\text { counter }\end{array}$ & 652 & 0.72 & $\begin{array}{c}2 \text { clock } \\
\text { cycles }\end{array}$ \\
\hline
\end{tabular}

\section{CONCLUSION}

In this paper we have masterminded a 2 bit, 3 bit \& 4 bit synchronous counters utilizing QCA progression \& saw a estimation of circuits which are checked utilizing QCADESIGNER gadget. In like manner with a few changes in a circuits we can watch n-bit synchronous counter \& barely any reasonable forms of 2 piece, 3 bits \& 4 bit counter.

\section{REFERENCES}

1. K. Kong, Y. Shang, \& R. Lu, "Counter designs in quantum-dot cellular automata," in Nanotechnology (IEEE-NANO), 2010 $10^{\text {th }}$ IEEE Conference on, pp. 1130-1134, aug. 2010.

2. Saifee H., Mehrotra A., Sarma R.: 'QCA based sequential \& combinational circuit design \& importance of parasitic components'.

3. First Int. Conf. Signal Propagation \& Computer Technology, ICSPCT 2014, July 12-13 2014, Ajmer, India, pp. 541-546
4. 3. Angizi S., Sayedsalehi S., Roohi A., ET AL.: 'Design \& verification of new n-bit quantum-dot synchronous counters using majority function-based JK flip-flops', J. Circuits Syst. Comput., 2015, 24, (10), pp. 1-16

5. 4. C. Lent, P. Tougaw \& W. Porod, "Quantum Cellular Automata: a physics of computing with arrays of quantum dot molecles", in physics \& computation, 1994. Phycomp'94, proceeding..workshop on pp. 5-13, Nov 1994.

6. 5. Lee Ai Lim, AzrulGhazali, Sarah Chan Tji Yan \& Chau Chine Fat "Sequential Circuit Design Using Quantum-Dot Cellular Automata (QCA)" IEEE Conf. Circuits \& Systems, pp. 162-167, October 2012.

7. A.O. Orlov, I. Amlani, G.H. Bernstein, C.S. Lent, G.L. Snider, "Realization of a Functional Cell for Quantum-Dot Cellular Automata", Science, Vol 277, pp 928-930, 1997.

8. Kim K., Wu K., Karri R.: 'Quantum-dot cellular automata design guideline', IEICE Trans. Fundam. Electron., Commun. Comput. Sci., 2006, E89-A, (6), pp. 1607-1614

Vankamamidi V., Ottavi M., Lombardi F.: 'Two-dimensional

9. schemes for clocking/timing of QCA circuits', IEEE Trans. Comput. Aided Des. Integr. Circuits Syst., 2008, 27, (1), pp. 34-44

\section{AUTHORS PROFILE}

E.Prashanthi, Assistant professor (ECE), Malla Reddy Engineering College for Women, Telanagana, India, prashanthiede@gmail.com

H. Bhagya Laxmi, Assistant professor (ECE), Malla Reddy Engineering College for Women, Telanagana, India,, Bhagya.hash@gmail.com 